\title{
True anteroposterior view pedicle screw insertion technique
}

\author{
This article was published in the following Dove Press journal: \\ Therapeutics and Clinical Risk Management \\ 29 June 2016 \\ Number of times this article has been viewed
}

Jia-yue Bai

Wei Zhang

Ji-long An

Ya-peng Sun

Wen-yuan Ding

Yong Shen

Key Biomechanical Laboratory of Orthopedics, The Third Hospital of Hebei Medical University, Shijiazhuang, Hebei, People's Republic of China
Correspondence: Wei Zhang Key Biomechanical Laboratory of Orthopedics, The Third Hospital of Hebei Medical University, 139 Ziqiang Road, Shijiazhuang 05005I, Hebei,

People's Republic of China

Tel +86 3II I8533II 2826

Email zwzw_zwei@I63.com
Background: The wide use of minimally invasive transforaminal lumbar interbody fusion (MIS-TLIF) surgery in the treatment of degenerative disc disease of lumbar spine in spinal surgery highlights the gradual decrease in the use of traditional pedicle screw insertion technology. This study aims to analyze the accuracy of the true anteroposterior view pedicle screw insertion technique in MIS-TLIF surgery, compare it with conventional pedicle screw insertion technology, and discuss its clinical application value.

Methods: Fifty-two patients undergoing true anteroposterior view (group A) and 87 patients undergoing conventional pedicle screw insertion (group B) were diagnosed with lumbar disc herniation or lumbar spinal stenosis. Time for screw placement, intraoperative irradiation exposure, accuracy rate of pedicle screw insertion, and incidence of neurovascular injury were compared between the two groups.

Results: The time for screw placement and intraoperative irradiation exposure was significantly less in group A. Penetration rates of the paries lateralis of vertebral pedicle, medial wall of vertebral pedicle, and anterior vertebral wall were $1.44 \%, 0 \%$, and $2.40 \%$, respectively, all of which were significantly lower than that in group B. No additional serious complications caused by the placement of screw were observed during the follow-up period in patients in group A, but two patients with medial penetration underwent revision for unbearable radicular pain.

Conclusion: The application of true anteroposterior view pedicle screw insertion technique in MIS-TLIF surgery shortens time for screw placement and reduces the intraoperative irradiation exposure along with a higher accuracy rate of screw placement, which makes it a safe, accurate, and efficient technique.

Keywords: true AP view, MIS-TLIF, pedicle screw, internal fixation, lumbar disc herniation

\section{Introduction}

Minimally invasive transforaminal lumbar interbody fusion (MIS-TLIF) is a minimally invasive spine surgical procedure that has been developed by combining with all types of expandable channels. After the introduction of MIS-TLIF by Foley et al, ${ }^{1}$ the procedure has been widely used in the treatment of lumbar instability and lumbar intervertebral disc disease and has become the most mature of minimally invasive spine surgery. ${ }^{2-4}$

Advances in minimally invasive surgery techniques for TLIF have reduced the incidence of complications and postoperative morbidity associated with conventional TLIF. ${ }^{5,6}$ When compared with conventional TLIF, MIS-TLIF appears to achieve similar fusion rates while reducing blood loss, soft tissue and muscle trauma, postoperative pain, and influence on spinal stabilization, increasing the speed of recovery. ${ }^{7-11}$ However, due to the small surgical incision and limited operating space, this technique, as with all minimally invasive surgical strategies, requires a steep learning curve that 
is associated with significantly longer X-ray exposure time and neural injury-related complications caused by lack of surgical skills proficiency. ${ }^{12,13}$ In MIS-TLIF surgery, percutaneous pedicle screw insertion is one of the core technologies for surgeons. Conventional anteroposterior (AP) and lateral $\mathrm{X}$-ray in standing position takes a long time for screw placement and higher time of intraoperative irradiation exposure, along with some errors. ${ }^{14}$ The phenomenon of pedicle screw misplacement occurs frequently, which can cause spinal cord or nerve injury, great vessels or viscera injury, dural matter tear and cerebrospinal fluid leak, pedicle fractures, or other complications in severely ill patients. Although computer navigation in surgery and other more accurate positioning technologies have been in use for some time in recent years, it brought about inherent high cost, learning curve, and other new problems to be resolved on the basis of solving old problems, seriously restricting the development and promotion of this technique.

This article focuses on the application of percutaneous pedicle screw insertion technique, true AP view pedicle screw insertion technique, to MIS-TLIF surgery, emphasizing the apparent benefits of this emerging technology. The technique can be achieved under the auxiliary of intraoperative AP C-arm fluoroscopic images. The accuracy and safety of this pedicle screw insertion technique were evaluated by observing the postoperative complications and computed tomography scan and comparing it with conventional pedicle screw insertion technique.

\section{Materials and methods Inclusion and exclusion criteria}

Inclusion criteria were: 1) patients preoperatively diagnosed with lumbar intervertebral disc protrusion with merger unilateral neurological symptoms and lumbar degenerative spondylolisthesis $\leq \mathrm{I}^{\circ} ; 2$ ) patients who do not recover after more than 3 months regular conservative treatment; and 3) patients who underwent single-segment MIS-TLIF.

Exclusion criteria were: 1) patients with previous spinal surgery, a history of malignancy, trauma, infection, severe osteoporosis, or congenital malformations; 2) patients preoperatively diagnosed with lumbar intervertebral disc protrusion with merger unilateral neurological symptoms and lumbar degenerative spondylolisthesis $>\mathrm{I}^{\circ}$; 3) patients who did not undergo MIS-TLIF surgery; 4) patients with other serious diseases and metal allergies; and 5) patients whose information is incomplete or who were out of touch during the follow-up period.

\section{Subjects and study design}

This study included 150 patients diagnosed with lumbar disc herniation or lumbar spinal stenosis, among which eleven patients were excluded because they were lost to follow-up; the remaining 139 patients underwent MIS-TLIF from February 2012 to December 2013. Seventy-seven males and 62 females (age range, 17-68 years; average age 51.9 years) were included. Appropriate subjects were selected according to the indication for MIS-TLIF surgery. According to the patient's will and randomized controlled principle, after approval by a votum of the Ethical Committee of The Third Hospital of Hebei Medical University, 52 patients who underwent true AP view and 87 patients who underwent conventional pedicle screw insertion were enrolled in this study. All patients were postoperatively followed-up for 7-29 months and the average follow-up for patients was 15.8 months. Lumbar AP and lateral X-rays films were obtained postoperatively, at 3 months postoperatively and at the time of the latest follow-up. Prior written informed consent was obtained from all of the subjects. This study was approved by the Ethical Committee of The Third Hospital of Hebei Medical University.

\section{Surgical techniques}

After routine preoperative preparation, following the induction of general endotracheal anesthesia, the patient was evenly placed in a prone position on a radiolucent operating table so as to maintain the physiologic lumbar lordosis. The MISTLIF procedure was performed on the symptomatic side. $\mathrm{C}$-arm guidance was used to determine the disc space and to draw the lateral pedicle line in the fluoroscopic anterior posterior view, and the lateral view was checked for tubular retractor system insertion. An incision was planned by connecting a line between the outer portions of both ends of the pedicles (Figure 1A and B). Then, a skin incision was made on the more symptomatic side or more severe pathologic side according to the images. The paravertebral muscles were split and retracted laterally to the outer edge of the facet joint. The lamina and facet joint were exposed by using monopolar cautery and pituitary forceps. After sequential dilatation, a tubular retractor system (METRx; Medtronic Sofamor Danek, Memphis, TN, USA) was docked on the facet joint.

Unilateral total facetectomy and laminectomy were carried out using a high-speed drill and osteotomes. Adequate decompression was achieved by cutting a portion of lamina and facet as autologous bone graft with an osteotome and rongeur. After a complete facetectomy, the ligamentum flavum was removed en bloc with a curette to expose dural sac 

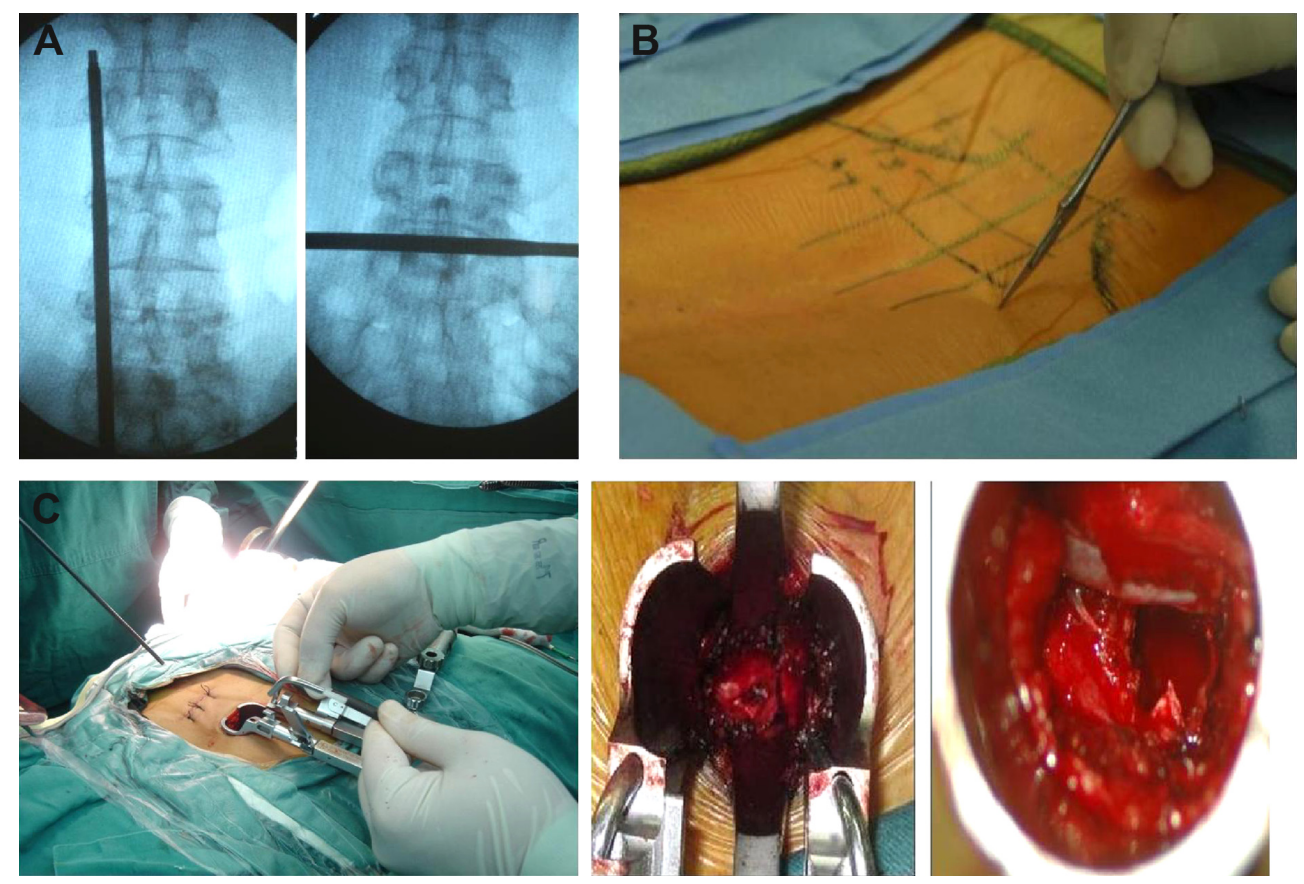

Figure I Preoperative localization and anterior cervical discectomy and fusion.

Notes: (A) The iliac crests were palpated preoperatively and then a mark was made on the overlying skin at the uppermost margin of each iliac crest. After placing the preoperative grid locator on the patient's back with the center in the midline over the approximate spinal levels of interest and fixing with an adhesive plaster, the AP fluoroscopic X-ray image involved in the sacrum was obtained. A metal bar was placed on the skin of patients in an axial (left panel) and lateral (right panel) direction for guiding the cut calibration. (B) The levels for surgery were confirmed according to the different markers on the locator and mark the incision and pedicles. (C) Decompression and bone grafting of facet joints aided by expanding channels. The operation field was exposed by expanding channels (left image) and the edges of vertebral plates and zygapophysial joints were observable (center image). The intervertebral disc and cartilage endplate were excised (right image).

Abbreviation: AP, anteroposterior.

and the lateral margin of the ipsilateral nerve root. To achieve decompression of the central canal and the contralateral side, the tubular retractor could be angled medially and the patient tilted laterally to decompress the contralateral side. Extensive decompression was performed, which included adequate decompression of central stenosis and the contralateral side. Then, the vertebral end plates were carefully prepared. Standard discectomy and endplate preparation were performed to construct the interbody fusion, and sufficient autologous bone graft obtained from the resected lamina and facet was packed in the anterior disc space. A single, long, bullet-shaped polyether-etherketone interbody cage (Capstone; Medtronic Sofamor Danek) filled with only autologous local bone was inserted obliquely across the disc space (Figure 1C). We did not perform any additional contralateral facet fusion in all the patients. After the interbody fusion was carried out, the tubular retractor was removed. The same decompression and fusion procedure were done at each segment. Following the decompression and fusion procedure, ipsilateral percutaneous pedicle screw was placed through the same skin incision, and contralateral percutaneous pedicle screw was also inserted through mirror incision under fluoroscopic guidance.

\section{Convention percutaneous pedicle screw insertion}

Depending on the depth of the tissue between the skin and pedicle, a 1-2 cm lateral skin incision was made to insert the Jamshidi needle at an appropriate angle into the pedicle, which was identified as a cylinder in adequate fluoroscopic images. Using the Jamshidi needle, docked against the bone at the junction of the base of the transverse process and facet joint, the lateral and AP C-arm fluoroscopic images were obtained to localize the needle tip and then the needle was gently tapped with a mallet to engage the tip in the bone. Fluoroscopy was intermittently used to confirm the direction and the depth to make sure the needle remained lateral to the medial pedicle wall. Under intermittent fluoroscopic guidance, the needle advanced to cross the pedicle center. The cannula was then exchanged for a flexible guidewire, which was inserted through the cannula into the pedicle. The fascia and muscle were dilated to allow screw placement. The pedicle was tapped using cannulated taps, and the percutaneous cannulated pedicle screw-rod system (Sextant; Medtronic Sofamor Danek) was placed. Titanium rod was used for bilateral compression screw fixation. 


\section{True AP view pedicle screw insertion}

$\mathrm{C}$-arm fluoroscopic images were obtained to localize the true AP when anterior and posterior edges of vertebral end plates overlapped in a line in the AP X-rays film. After the Quadrant system was removed, pedicle entry point was directly touched through the surgeon's index finger within the incision. The skin incision, typically $2-2.5 \mathrm{~cm}$ in length, is centered on the spinal needle entry point, parallel to the midline and $\sim 4-5 \mathrm{~cm}$ lateral to it. After local hemostasis for the skin edges, the incision of the subcutaneous fat and lumbar fascia is continued with the 10-blade in a lateral to medial direction, and maintaining the same cranial to caudal angulation as the localizing spinal needle. The paraspinous muscle dissection was performed with the index finger, or with one of the smaller tubular dilators if the finger cannot fit through the skin incision. Lateral fluoroscopy was used to confirm the bony landmark to be identified with either the finger or the dilator, which is the junction between the spinous process and the lamina of the level of interest (eg, the L3 lamina if the L3-4 fusion is to be performed). Then, the paraspinous muscles can be gently detached from the underlying lamina with the tubular dilator. The tubular dilators of increasing size are then used to insert the final tubular retractor of $\sim 18-20 \mathrm{~mm}$ (Figure 2A). The correct placement of the tube is the 3 and 9 o'clock positions of bilateral vertebral pedicle, which is confirmed with AP fluoroscopy (Figure 2B and C). After the Jamshidi needle was advanced through the pedicle into the vertebral body under fluoroscopic guidance, a blunt-tipped guidewire was inserted into the screw path. The appropriately sized cannulated pedicle screws were placed along the guidewire into the pedicle under fluoroscopic guidance using the percutaneous cannulated pedicle screw-rod system as mentioned above. Titanium rod was used for bilateral compression screw fixation.

No active bleeding of incision was noted. Drainage tube was placed and then the incision was sutured layer by layer. Three experienced spinal chief physicians from major hospitals participated in the surgical operation.

\section{Clinical, radiological, and follow-up evaluation}

\section{Time for pedicle screw insertion}

From the beginning of pedicle screw placement after operative decompression to the end pedicle screw placement, time for screw placement of the two percutaneous pedicle screw insertion techniques were counted, respectively. The average time required for placement of each screw and the proportion of time for screw placement to total operative time were recorded and computed.

\section{Intraoperative irradiation exposure}

Intraoperative irradiation exposures of the two percutaneous pedicle screw insertion techniques were measured and compared.

\section{The accuracy of pedicle screw insertion}

Computed tomography of vertebral pedicle of the operative segment was performed on all patients after surgery. The percentage of pedicle screws that penetrate into the paries lateralis of vertebral pedicle, medial wall of vertebral pedicle, and anterior vertebral wall in the two percutaneous pedicle screw insertion techniques were calculated and compared.
A

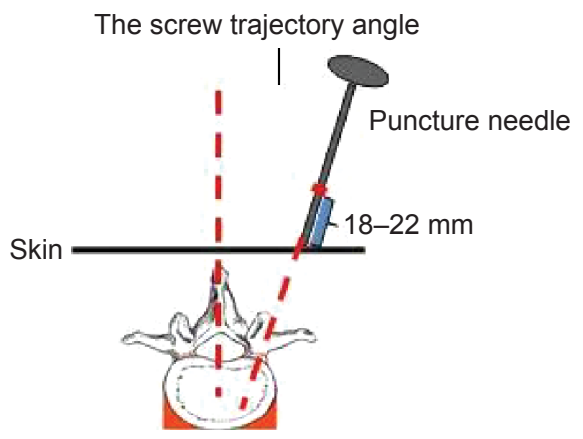

B

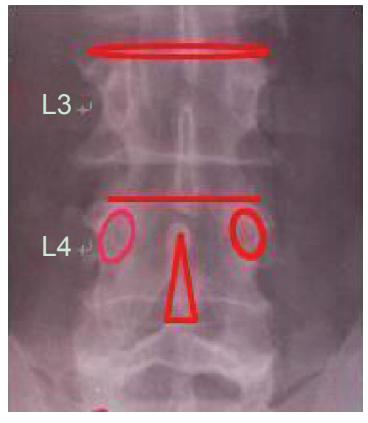

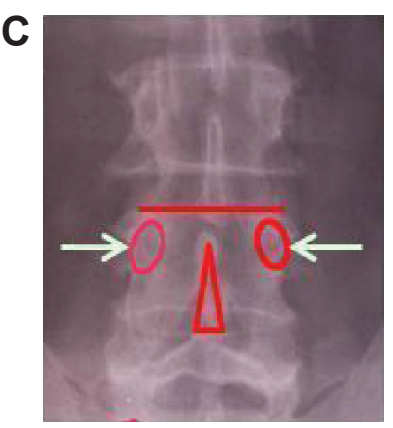

Figure 2 True AP view percutaneous pedicle screw insertion and clinical and radiological evaluation.

Notes: (A) The pedicular convergent angle and screw trajectory angle were measured. The tubular dilators of increasing size were used to insert the final tubular retractor of 18-20 mm. (B) Intraoperative radiograph showing the localizing spinal needle inserted in perfect alignment with the targeted L3 and L4 disc. C-arm fluoroscopic images were obtained to localize the true AP, when the anterior and posterior edges of vertebral end plates at L4 disc overlap in a line. (C) The correct placement of the tube is the 3 and 9 o'clock positions of bilateral vertebral pedicle (arrows).

Abbreviation: AP, anteroposterior. 


\section{The incidence of neurovascular injury complications}

The incidence of neurovascular injury complications related to pedicle screw misplacement in the two percutaneous pedicle screw insertion techniques were analyzed and compared.

\section{Statistical analysis}

Statistical analysis was performed with SPSS Version 13.0 (SPSS Inc., Chicago, IL, USA). All measurement data were analyzed by normality test and homogeneity test of variances. All data were recorded as mean \pm standard deviation. Student's $t$-test and Fisher's exact test were used for intragroup comparison between the preoperative and postoperative state in each group. In all analyses, significance was defined as $P<0.05$.

\section{Results}

A total of 208 pedicle screws were inserted in group A and 348 pedicle screws were placed in group B. Postoperation follow-up X-ray films of all patients revealed that graft fusion was achieved in operative levels of patients at the time of the last follow-up.
Operative data for conventional and true AP view percutaneous pedicle screw insertion surgical procedures are presented in Figure 3. The time for screw placement was 4.7 minutes for conventional and 8.4 minutes for true AP view percutaneous pedicle screw insertion surgical procedures. There was statistically significant difference found in the mean time for screw placement (Figure 3A, $P<0.05$, Student's $t$-test). In addition, the intraoperative irradiation exposure was significantly lower for true AP view percutaneous pedicle screw insertion compared to conventional percutaneous pedicle screw insertion, with an average of 5.6 seconds in group A and 14.9 seconds in group B (Figure 3B, $P<0.05$ ).

Moreover, among the 556 pedicle screws interpreted in this series, $500(89.93 \%)$ screws were interpreted as being correctly inserted. Postoperative radiographs showed three (1.44\%) screws in group A and 24 (6.90\%) screws in group B were overlong and penetrating into the paries lateralis of vertebral pedicle. Among the frank penetrations, ten (2.87\%) screws in group B were found to penetrate into the medial wall of vertebral pedicle, whereas there was no screw violation
A

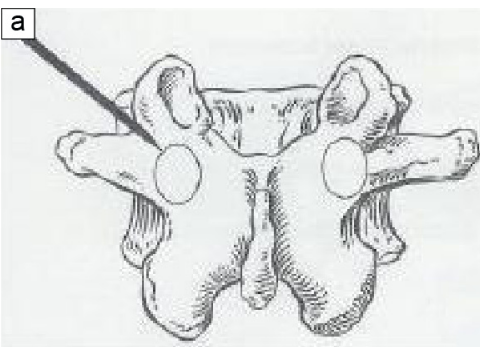

B

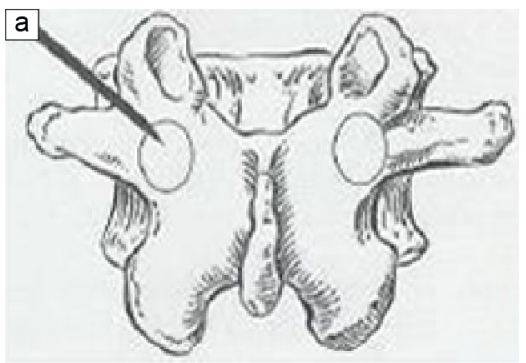

C

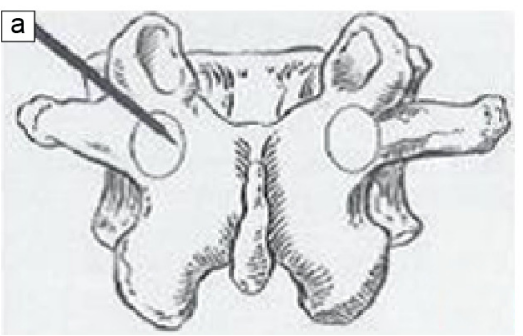

b

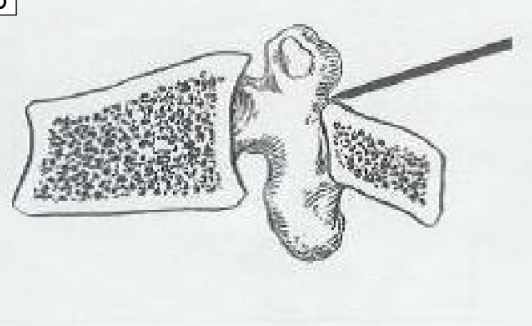

b

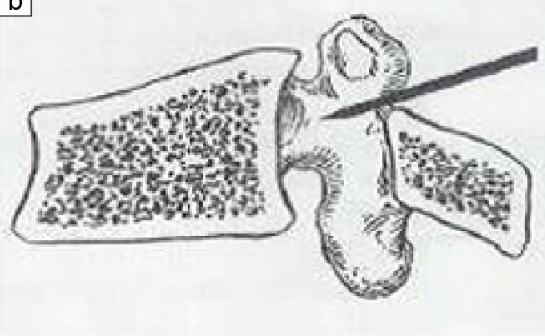

b

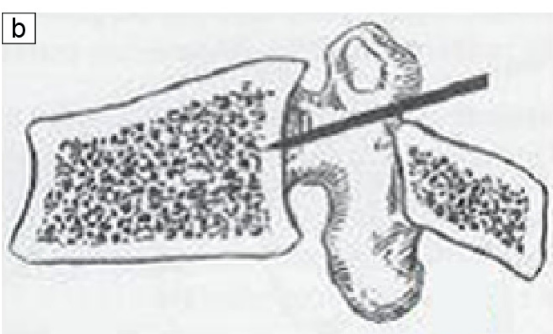

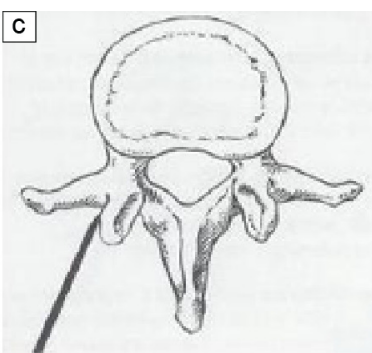

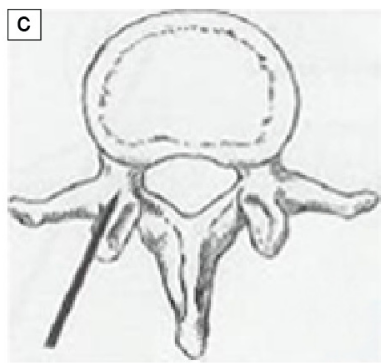

C

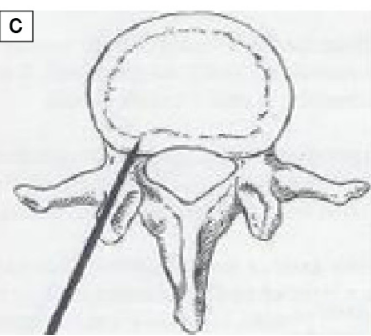

Figure 3 Diagrams for pedicle screw insertion.

Notes: (A) Diagram for initialized position of pedicle screw insertion: a) anteroposterior, b) lateral, and c) axial. (B) Diagram for interposition of pedicle screw insertion: a) anteroposterior, b) lateral, and c) axial. (C) Diagram for security bit of pedicle screw insertion: a) anteroposterior, b) lateral, and c) axial. 
Table I Comparison of the accuracy rate during insertion of pedicle screws between groups $A$ and $B$

\begin{tabular}{llll}
\hline Parameters & $\begin{array}{l}\text { Group A } \\
(\mathbf{n}=\mathbf{2 0 8})\end{array}$ & $\begin{array}{l}\text { Group B } \\
(\mathbf{n}=\mathbf{3 4 8})\end{array}$ & P-value \\
\hline $\begin{array}{l}\text { Penetration rate of the paries } \\
\text { lateralis of vertebral pedicle \% }\end{array}$ & 1.44 & 6.90 & 0.00 \\
$\begin{array}{l}\text { Penetration rate of the medial } \\
\text { wall of vertebral pedicle \% }\end{array}$ & 0 & 2.87 & 0.02 \\
$\begin{array}{l}\text { Penetration rate of the } \\
\text { anterior vertebral wall \% }\end{array}$ & 2.4 & 10.34 & 0.00 \\
$\begin{array}{l}\text { The accuracy rate during } \\
\text { insertion of pedicle screws \% }\end{array}$ & $96.6^{\mathrm{a}}$ & $83.05^{\mathrm{b}}$ & 0.00 \\
\hline
\end{tabular}

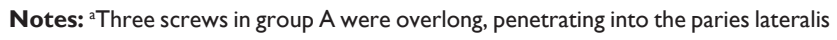
of vertebral pedicle, among which one screw also penetrated into the anterior vertebral wall. ${ }^{\text {} T}$ Twenty-four screws in group $B$ were overlong, penetrating into the paries lateralis of vertebral pedicle, among which eleven screws also penetrated into the anterior vertebral wall. Group A: patients undergoing true anteroposterior view. Group B: patients undergoing conventional pedicle screw insertion.

in the medial wall of vertebral pedicle in group A. Besides, fracturing of the anterior vertebral wall caused by five (2.40\%) crews in group A and 55 (10.34\%) crews in group B was observed. Comparison results of the two groups showed that the penetration rate in group A was significantly lower than that in group $\mathrm{B}$ (Table $1, P<0.05$ ). No additional serious complications caused by the placement of screw were observed during the follow-up period in patients in group A. However, two patients with medial penetration underwent revision for unbearable radicular pain. The placement of screw in the patient with unbearable radicular pain was judged to show moderate medial misplacement; the patients recovered completely after repositioning of the screw on the following day (Figure 3C). The complication rate was $0.57 \%$.

\section{Discussion}

The application of pedicle screw fixation in spine surgery has a history of more than 50 years. Posterior transpedicular screw initially was reported by Boucher in 1959, which was used for lumbosacral fusion fixation and gained good results. ${ }^{15}$ Since then, various pedicle screw fixation systems have been used widely in spine surgery, which have strongly promoted the development of spine surgery ${ }^{16}$ Recently, with the popularity of minimally invasive concept, a variety of minimally invasive spine surgery techniques arise at the historic moment. MIS-TLIF was also widely accepted and quickly spread because of its minimal trauma, less complications, and steady curative effect. ${ }^{2-4}$ Recently, the concept of MIS-TLIF has been widely recognized, and a variety of minimal invasion spine surgery techniques have been rapidly developed. As an alternative technology to traditional open TLIF, percutaneous pedicle screw insertion has the potential benefit of minimized surgical incision and tissue disruption, less paravertebral muscles damage, and reduced blood loss and length of hospitalization. ${ }^{17}$ Most scholars believe that percutaneous placement of pedicle screws can reduce related complications, which is a safe and effective approach. ${ }^{14,18}$

MIS-TLIF offers several potential advantages, including postoperative back pain and leg pain, intraoperative blood loss, transfusion, and duration of hospital stay postoperatively in treating two-level lumbar degenerative disease, but other studies reported disadvantages of MIS-TLIF, such as steep learning curve and incomplete decompression. ${ }^{19,20}$ Literatures reported it required much more radiation exposure. ${ }^{4,21}$ In addition, as for the accuracy of pedicle screw insertion, previous studies found that though the incidence rate of screw misplacement during percutaneous pedicle screw insertion is lower than traditional open surgery, an incidence rate of $6 \%-23 \%$ still exists. ${ }^{14,22-24}$ To improve the accuracy of pedicle screw insertion and reduce intraoperative irradiation exposure, researchers have proposed all sorts of means for intraoperative monitoring screw placement, particularly for recent application of computer-aided technology that can significantly reduce the incidence of screw misplacement. ${ }^{25,26}$ But computer-aided technology required high-tech equipment and expensive titanium alloy implants, which is difficult to be popularized.

True AP view pedicle screw insertion technique in this study is based on anatomic characteristics of vertebral pedicle and develops from conventional pedicle screw insertion. Usually, pedicle length, that is the length from entrance point of pedicle screw placement to vertebral posterior, is $\sim 14.6-19.13 \mathrm{~mm} .{ }^{27,28}$ Thus, accurate pedicle screw insertion process can be divided into initialized position, interposition, and security bit. When in initialized position, puncture needle was at the 3 and 9 o'clock position of vertebral pedicle in AP X-ray view and behind the pedicle in lateral X-ray film (Figure 4A). When in interposition, puncture needle was at the neutral position of vertebral pedicle in AP X-ray film and half into the pedicle in lateral X-ray film (Figure 4B). When in security bit, puncture needle was at the inner margin of vertebral pedicle in AP X-ray film and completely into the pedicle to vertebral posterior in lateral X-ray film (Figure 4C). The development of true AP view percutaneous pedicle screw insertion technique is based on the analysis of corresponding relation of AP and lateral X-ray films in the accurate screw placement process. The key of this technique is to ensure that the $\mathrm{C}$-arm fluoroscopy is located in the true AP position of transpedicular vertebral body, which means that the anterior and posterior edges of vertebral endplates overlap in a line, bilateral pedicle screw presents positional symmetry with closing to the upper end plate, and spinous process is located 

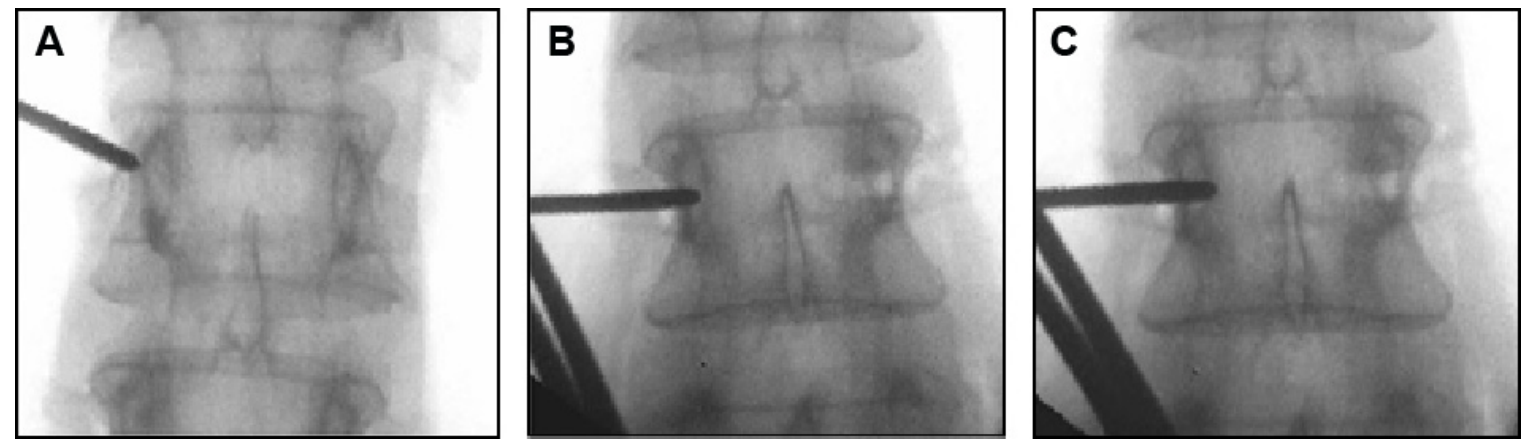

Figure $4 \mathrm{X}$-ray films of true AP view percutaneous pedicle screw insertion.

Notes: (A) Initialized position. (B) Inserting the needles $10 \mathrm{~mm}$ in interposition. (C) Inserting the needles 20 mm in security bit.

Abbreviation: AP, anteroposterior.

in the central vertebral body with closing to the lower end plate. It is based on true AP X-ray view and the direction of the needle is kept parallel to upper end plate - keep abduction angle measured in axial view, pinpoint entrance point of pedicle screw placement in initialized position, and then insert needle $18-20 \mathrm{~mm}$ into the security bit through interposition. It does not need intraoperative fluoroscopy of lateral X-ray and only needs X-ray confirmation of true AP (Figure 5).

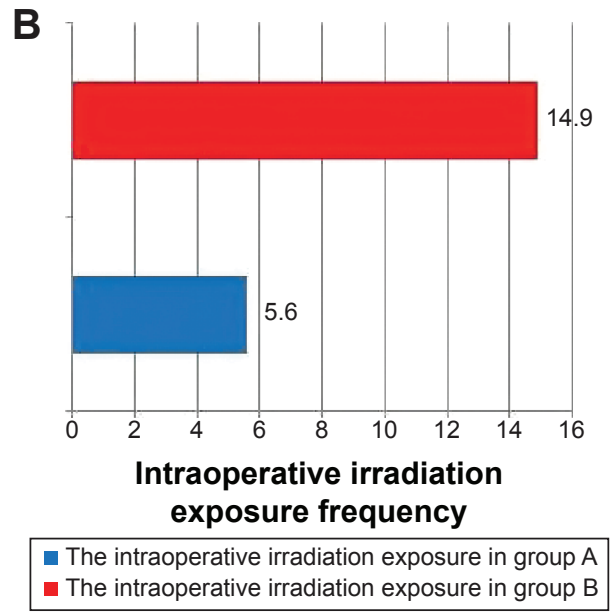

- The intraoperative irradiation exposure in group B

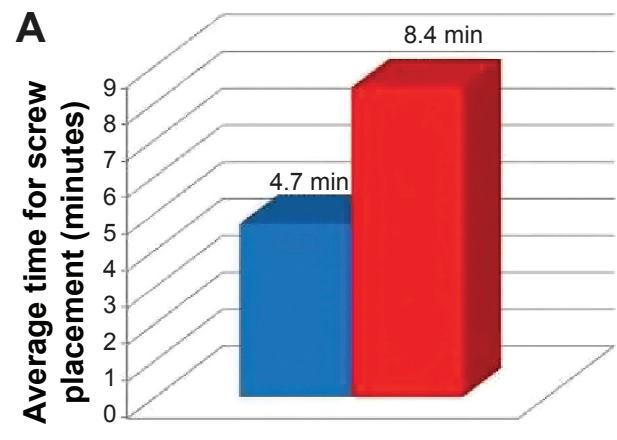

Average time for screw placement in group $\mathrm{A}$
Average time for screw placement in group B

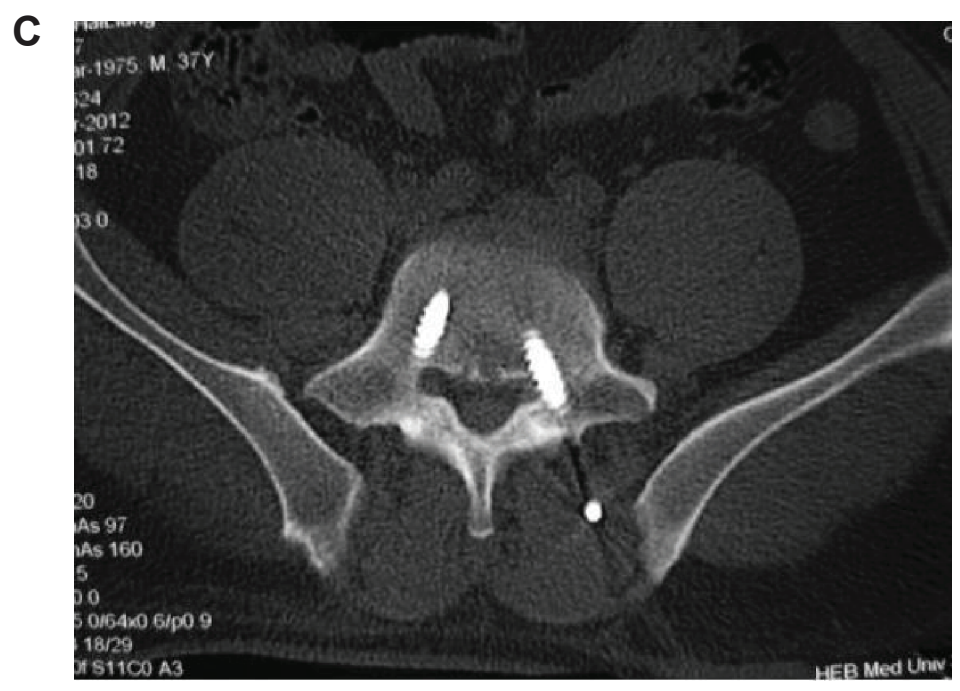

Figure 5 Clinical and radiological evaluation.

Notes: (A) The mean time for screw placement during true AP view percutaneous pedicle screw insertion was significantly less than that during conventional percutaneous pedicle screw insertion. (B) The intraoperative irradiation exposure for true AP view percutaneous pedicle screw insertion was significantly lower than conventional percutaneous pedicle screw insertion. (C) Postoperative X-ray and CT of vertebral pedicle after MIS-TLIF surgery showed that a 37 -year-old male presented with the left pedicle screw misplacement at L5 disc and nerve root compression.

Abbreviations: AP, anteroposterior; CT, computed tomography; MIS-TLIF, minimally invasive transforaminal lumbar interbody fusion. 
If puncture needle enters $18-20 \mathrm{~mm}$ through the initialized position and still does not penetrate into the inner edge of vertebral pedicle in true AP X-ray film, the accuracy and security of pedicle screw placement could be guaranteed. On the other hand, if puncture needle penetrates into the inner edge of vertebral pedicle in true AP X-ray film before entering $20 \mathrm{~mm}$, we can speculate that the puncture needle enters into spinal canal.

The study is designed to compare patients who underwent true AP view pedicle screw insertion technique and patients who underwent conventional percutaneous pedicle screw insertion technique. Results demonstrated that compared to conventional percutaneous pedicle screw insertion technique, the remarkable advantages of true AP view pedicle screw insertion technique are as follows: 1) it reduces time for screw placement (Figure 3A). The application of true AP view pedicle screw insertion technique only needs the auxiliary of AP X-ray view and does not have to adjust the C-arm to lateral X-ray view. Furthermore, true AP X-ray film of intraoperative transpedicular vertebral body is not affected by vertebral side convex, vertebral rotation, and other abnormal location of vertebral body. It can provide absolute position reference for direction during the screw fixation and thus reduces time for screw placement and improves the efficiency of screw placement. 2) It reduces intraoperative irradiation exposure (Figure 3B). True AP view pedicle screw insertion technique was applied using only AP X-ray view to deduce the depth of puncture needle into vertebral pedicle in lateral view, without the auxiliary of lateral X-ray view, which could remarkably reduce intraoperative irradiation exposure. 3) It improves the accuracy of pedicle screw insertion (Table 1). A total of 208 pedicle screws were inserted in patients undergoing true AP view pedicle screw insertion technique. Postoperative computed tomography of vertebral pedicle showed that there were three screws penetrating into the paries lateralis of vertebral pedicle, five screws penetrating into the anterior vertebral wall, and no screws penetrating into the medial wall of vertebral pedicle. Compared to 348 pedicle screws placed in patients undergoing conventional pedicle screw insertion technique, true AP view pedicle screw insertion technique showed a significantly higher accuracy of screw placement. What is more, since the application of true AP view pedicle screw insertion technique only needs the auxiliary of AP $\mathrm{X}$-ray view and does not have to adjust the $\mathrm{C}$-arm to lateral $\mathrm{X}$-ray view, it may theoretically reduce the risk of perioperative infection. The accuracy of true AP view pedicle screw insertion technique was $96.6 \%$ in the current study, which has no obvious advantage when compared to computer navigation surgery, ${ }^{21}$ but just the auxiliary of C-arm fluoroscopy is needed for true AP view pedicle screw insertion technique, which is obviously superior to computer navigation surgery in terms of required equipment, operation cost, and popularity. If surgeons have a good command of this technique, the accuracy of pedicle screw insertion may be remarkably improved. Besides, the statistical data in the present study were from one-man operated results. Therefore, if the assistants expertly master this technique as well, time for screw placement could be further shortened and the intraoperative irradiation exposure could be further reduced.

\section{Conclusion}

The application of true AP view pedicle screw insertion technique in MIS-TLIF surgery reduces the time for screw placement and the intraoperative irradiation exposure along with higher accuracy rate of screw placement, which is a safe, accurate, and efficient percutaneous pedicle screw insertion technique.

\section{Disclosure}

The authors report no conflicts of interest in this work.

\section{References}

1. Foley KT, Holly LT, Schwender JD. Minimally invasive lumbar fusion. Spine. 2003;28:S26-S35.

2. Mummaneni PV, Rodts GE Jr. The mini-open transforaminal lumbar interbody fusion. Neurosurgery. 2005;57:256-261; discussion 256-261.

3. Ozgur BM, Hughes SA, Baird LC, Taylor WR. Minimally disruptive decompression and transforaminal lumbar interbody fusion. Spine J. 2006; 6:27-33.

4. Gu G, Zhang H, Fan G, et al. Comparison of minimally invasive versus open transforaminal lumbar interbody fusion in two-level degenerative lumbar disease. Int Orthop. 2014;38:817-824.

5. Foley KT, Gupta SK. Percutaneous pedicle screw fixation of the lumbar spine: preliminary clinical results. J Neurosurg. 2002;97:7-12.

6. Foley KT, Lefkowitz MA. Advances in minimally invasive spine surgery. Clin Neurosurg. 2002;49:499-517.

7. Schizas C, Tzinieris N, Tsiridis E, Kosmopoulos V. Minimally invasive versus open transforaminal lumbar interbody fusion: evaluating initial experience. Int Orthop. 2009;33:1683-1688.

8. Peng CW, Yue WM, Poh SY, Yeo W, Tan SB. Clinical and radiological outcomes of minimally invasive versus open transforaminal lumbar interbody fusion. Spine (Phila Pa 1976). 2009;34:1385-1389.

9. Tian NF, Wu YS, Zhang XL, Xu HZ, Chi YL, Mao FM. Minimally invasive versus open transforaminal lumbar interbody fusion: a meta-analysis based on the current evidence. Eur Spine J. 2013;22:1741-1749.

10. Perez-Cruet MJ, Hussain NS, White GZ, et al. Quality-of-life outcomes with minimally invasive transforaminal lumbar interbody fusion based on long-term analysis of 304 consecutive patients. Spine. 2014;39: E191-E198.

11. Seng C, Siddiqui MA, Wong KP, et al. Five-year outcomes of minimally invasive versus open transforaminal lumbar interbody fusion: a matched-pair comparison study. Spine. 2013;38:2049-2055. 
12. Villavicencio AT, Burneikiene S, Roeca CM, Nelson EL, Mason A. Minimally invasive versus open transforaminal lumbar interbody fusion. Surg Neurol Int. 2010;1:12.

13. Silva PS, Pereira P, Monteiro P, Silva PA, Vaz R. Learning curve and complications of minimally invasive transforaminal lumbar interbody fusion. Neurosurg Focus. 2013;35:E7.

14. Raley DA, Mobbs RJ. Retrospective computed tomography scan analysis of percutaneously inserted pedicle screws for posterior transpedicular stabilization of the thoracic and lumbar spine: accuracy and complication rates. Spine. 2012;37:1092-1100.

15. Boucher HH. A method of spinal fusion. J Bone Joint Surg Br. 1959; 41-B:248-259.

16. Kasten MD, Rao LA, Priest B. Long-term results of iliac wing fixation below extensive fusions in ambulatory adult patients with spinal disorders. J Spinal Disord Tech. 2010;23:e37-e42.

17. Dahdaleh NS, Smith ZA, Hitchon PW. Percutaneous pedicle screw fixation for thoracolumbar fractures. Neurosurg Clin N Am. 2014;25: 337-346.

18. Kim MC, Chung HT, Cho JL, Kim DJ, Chung NS. Factors affecting the accurate placement of percutaneous pedicle screws during minimally invasive transforaminal lumbar interbody fusion. Eur Spine J. 2011; 20:1635-1643

19. Jin-Tao Q, Yu T, Mei W, et al. Comparison of MIS vs. open PLIF/TLIF with regard to clinical improvement, fusion rate, and incidence of major complication: a meta-analysis. Eur Spine J. 2015;24:1058-1065.

20. Hey HW, Hee HT. Lumbar degenerative spinal deformity: Surgical options of PLIF, TLIF and MI-TLIF. Indian J Orthop. 2010;44(2): 159-162.
21. Funao H, Ishii K, Momoshima S, et al. Surgeons' exposure to radiation in single- and multi-level minimally invasive transforaminal lumbar interbody fusion; a prospective study. PLoS One. 2014;9:e95233.

22. Wiesner L, Kothe R, Ruther W. Anatomic evaluation of two different techniques for the percutaneous insertion of pedicle screws in the lumbar spine. Spine. 1999;24:1599-1603.

23. Wiesner L, Kothe R, Schulitz KP, Ruther W. Clinical evaluation and computed tomography scan analysis of screw tracts after percutaneous insertion of pedicle screws in the lumbar spine. Spine. 2000;25: 615-621.

24. Schizas C, Michel J, Kosmopoulos V, Theumann N. Computer tomography assessment of pedicle screw insertion in percutaneous posterior transpedicular stabilization. Eur Spine J. 2007;16:613-617.

25. Amiot LP, Lang K, Putzier M, Zippel H, Labelle H. Comparative results between conventional and computer-assisted pedicle screw installation in the thoracic, lumbar, and sacral spine. Spine. 2000;25:606-614.

26. Youkilis AS, Quint DJ, McGillicuddy JE, Papadopoulos SM. Stereotactic navigation for placement of pedicle screws in the thoracic spine. Neurosurgery. 2001;48:771-778; discussion 778-779.

27. Hou S, Hu R, Shi Y. Pedicle morphology of the lower thoracic and lumbar spine in a Chinese population. Spine(Phila Pa 1976). 1993;18: $1850-1855$.

28. Kadioglu HH, Takci E, Levent A, Arik M, Aydin IH. Measurements of the lumbar pedicles in the Eastern Anatolian population. Surg Radiol Anat. 2003;25:120-126.
Therapeutics and Clinical Risk Management

\section{Publish your work in this journal}

Therapeutics and Clinical Risk Management is an international, peerreviewed journal of clinical therapeutics and risk management, focusing on concise rapid reporting of clinical studies in all therapeutic areas, outcomes, safety, and programs for the effective, safe, and sustained use of medicines. This journal is indexed on PubMed Central, CAS,

\section{Dovepress}

EMBase, Scopus and the Elsevier Bibliographic databases. The manuscript management system is completely online and includes a very quick and fair peer-review system, which is all easy to use. Visit http://www.dovepress.com/testimonials.php to read real quotes from published authors.

Submit your manuscript here: http://www.dovepress.com/therapeutics-and-clinical-risk-management-journal 